\title{
Praxis unter Wasser? Was nun?
}

\section{Die Praxisversicherung für alle Fälle}

Montagmorgen nach einem erholsamen Wochenende. Es erwartet Sie eine neue Woche, die mit Patiententerminen gefüllt ist. Was für eine Überraschung, als Sie Ihre Praxis betreten. Die gesamten Räumlichkeiten stehen unter Wasser. Wie sollen Sie nun weiterarbeiten?

\section{Die FMH-Insurance-Services-Praxisversicherung hilft}

Hoffentlich haben Sie sich für die FMH-Insurance-Services-Praxisversicherung entschieden. Ein Telefonanruf genügt, und die nötigen Schritte werden eingeleitet. Die Versicherung deckt Schäden, die durch Feuer, Elementarereignisse (z. B. Überschwemmung, Lawine, Felssturz usw.), Wasser, Einbruchdiebstahl oder Beraubung entstanden sind. Darüber hinaus kann auch der Umsatzverlust mitversichert werden, der durch diese Ereignisse entstehen kann.

\section{Bedürfnisgerechte Versicherungslösung}

Je nach Spezialisierung einer Praxis sind auch die Versicherungsbedürfnisse unterschiedlich. Die FMHInsurance-Services-Praxisversicherung ist modular aufgebaut und bietet die nötige Flexibilität. Verschiedene Zusatzdeckungen wie z.B. die Versicherung medizinischer Geräte oder eines Notfallkoffers können wahlweise eingeschlossen werden.

\section{Attraktive Prämie für FMH-Mitglieder}

Die FMH-Insurance-Services-Praxisversicherung bietet ein optimales Preis-Leistungs-Verhältnis. Marktvergleiche haben gezeigt, dass unsere Prämien gegenüber der Konkurrenz bis zu $40 \%$ tiefer sind.

\section{Profitieren Sie jetzt und bestellen Sie eine persönliche Offerte}

Ihre Bedürfnisse stehen bei uns im Mittelpunkt. Mit unserem Namen garantieren wir Ihnen die Betreuung durch professionelle Berater mit langjähriger Erfahrung im Versicherungs- und Bankenbereich. Dank unserer Unabhängigkeit können wir Ihnen das für Sie am besten geeignete Produkt anbieten.

\section{Antworttalon}

Vorname / Name

Adresse

PLZ / Ort

\section{Geburtsdatum}

Telefon Privat/Geschäft

Beste Zeit für einen Anruf

Bitte senden Sie mir eine Offerte zur FMH-Insurance-Services-Praxisversicherung (bitte eine aktuelle Policenkopie beilegen).

O Ich wünsche eine persönliche Beratung. Bitte rufen Sie mich an.

Ich interessiere mich für folgende Produkte:

\begin{tabular}{ll} 
Krankenkasse & Pensionskasse BVG \\
Säule $3 a$ & $\bigcirc$ Rechtsschutzversicherung \\
Finanz-/Steuerplanung & $\bigcirc$ Berufshaftpflichtversicherung \\
\hline
\end{tabular}

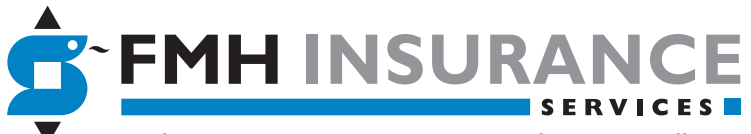

Roth Gygax \& Partner AG $\square$ Koordinationsstelle Moosstrasse 2 『 3073 Gümligen

Telefon 0319595000 = Fax 0319595010 mail@fmhinsurance.ch $\square$ www.fmhinsurance.ch 